\title{
Urgences
}

\section{Américanité et Amérique}

\section{Maximilien Laroche}

Numéro 34, décembre 1991

Mythes et Romans de l’Amérique

URI : https://id.erudit.org/iderudit/025687ar

DOI : https://doi.org/10.7202/025687ar

Aller au sommaire du numéro

Éditeur(s)

Urgences

ISSN

0226-9554 (imprimé)

1927-3924 (numérique)

Découvrir la revue

Citer cet article

Laroche, M. (1991). Américanité et Amérique. Urgences, (34).

https://doi.org/10.7202/025687ar d'utilisation que vous pouvez consulter en ligne.

https://apropos.erudit.org/fr/usagers/politique-dutilisation/ 


\section{Américanité et Amérique}

\section{Maximilien Laroche}

On parle beaucoup d'américanité, ces temps-ci. Beaucoup moins d'Amérique et pas du tout d'américanisation. Il faudrait, me semble-t-il, inverser la vapeur, ou du moins passer à une autre vitesse de réflexion. Et pour commencer parler un peu plus de l'Amérique ou plus exactement des Amériques.

C'est par un coup de force linguistique, du genre des occupations militaires de certains pays par les U.S. Marines, que les citoyens des États-Unis se sont approprié le nom d'Américains. Ils ne sont ni plus ni moins que des ÉtatsUniens comme les Mexicains, les Haïtiens, les Canadiens, lesquels sont tous, et chacun à part entière, Américains puisque habitants du continent américain.

Mais peut-être qu'il faudrait, au-delà de cette question juridico-sémantique, en venir un jour à examiner ce paradoxe qui fait confirmer par la pratique ce que la théorie ne peut que nier.

Les États-Unis d'Amérique du Nord (U.S.A.). sont sans doute l'exemple le plus accompli d'un rêve américain et c'est à ce titre peut-être qu'ils prétendent être l'Amérique ou du moins la seule Amérique viable et valable. Et il faut reconnaître que cette prétention semble accréditée aux yeux non seulement de l'ensemble des peuples américains mais du monde entier.

En tout cas les courants migratoires qui emportent vers la terre de l'Oncle Sam les Mexicains des zones frontalières, les Cubains, les Haïtiens et les Dominicains de la côte caraibe, sans parler des vagues antérieures d'immigrants qui venaient d'Europe ou d'Asie, semblent confirmer qu'aux yeux de pas mal de gens, et de par la terre entière, les U.S.A. sont bien l'incarnation de l'Amérique idéale.

Il n'en demeure pas moins que les États-Unis ne sont qu'une des Amériques réelles ou possibles. Et c'est sans doute pour cela que l'américanité entendue au sens "d'états-unité " fait problème et est un mot de sens ambigu. 
Peut-être même que ce n'est pas seulement à cause de cette appropriation, confiscation et monopolisation indue, de cette privatisation en fin de compte d'un terme collectif que le mot américanité fait problème. Les États-Unis, comme n'importe quel autre pays d'ailleurs, peuvent et doivent être remis en question. Et de fait, ils ne manquent pas de l'être, comme par exemple dans un récent numéro des Cahiers de recherche sociologique ${ }^{1}$.

Pourtant quand il s'agit de cinéma, le volume de la production états-unienne faisant sans doute écran à la clarté terminologique, on prend volontiers la production hollywoodienne pour celle de toute l'Amérique. Et la revue Cinémas ${ }^{2}$ peut donc consacrer un numéro au thème suivant: Américanité et cinéma qui ne considère que la seule cinématographie états-unienne. Du moins celle-ci est-elle prise comme le parangon de l'expression de l'américanité. Et même si quelques collaborateurs parlent soit d'un cinéaste allemand, Wim Wenders, soit d'un français, Jean Renoir, soit d'un québécois, Denys Arcand (Le déclin de l'empire américain oblige!), c'est toujours par référence au cinéma d'Hollywood. Le titre de l'article de Roger Viry-Babel est d'ailleurs éloquent: «Jean Renoir à Hollywood ou la recherche américaine d'une image française". Pour chercher l'américanité, il suffit de se rendre à Hollywood, semble-t-il.

Pourtant ces mêmes collaborateurs ne manquent pas de souligner l'ambiguïté du mot américanité. D'entrée de jeu, dès le mot de présentation, Martin Lefebvre le déclare: * Limportance de l'américanité dans la culture contemporaine ne fait aucun doute. Pourtant plusieurs interrogations subsistent quant à sa nature: s'agit-il d'une forme symbolique, d'un mythe moderne ou d'une idéologie?" Mais il poursuit: "Le fameux american way of life a restructuré les besoins de la société postindustrielle - on mange maintenant des Big Macs à Moscou. ${ }^{3}$ Et voilà l'américanité, encore une fois confondue avec l'états-unité.

1 Cahiers de recherche sociologique, « Les États-Unis en question ", n $n^{0} 15$, automne 1990. Montréal, UQAM.

2 Cinéma, revue d'études cinématographiques/Journal of Film Studies, Américanité et cinéma, vol I, n* 1-2, automne 1990.

3 Martin Lefebvre, * Présentation ", Cinémas, ibid., p. 8. 
90

Heinz Weinmann, quant à lui, fait l'analyse d'un film de D.W. Griffith dont le titre même: Birth of a nation, est fort clair quant à son sujet: la naissance d'une nation parmi d'autres: les U.S.A. Weinmann démonte même dans le syntagme nominal * United States of America * le mécanisme d'une opération idéologique: *L'indien, son nom est une dénégation, un refoulement, voulant dire que sa place n'est pas sur ce continent. Les Américains, ce sont les Blancs. Même plus besoin de Conquête. " Mais plus profondément, cette première désorientation de tout le continent américain devient l'orientation même de la nation anglo-saxonnne qui s'appellera, dès le 19 juillet 1776, United States of America. Avec la naissance de la nation américaine, cette dernière utilise bien l'article partitif dans son nom officiel * of America * signifiant que ce pays est une partie d'un tout global - continent américain -, mais l'habitant s'appellera dorénavant "Américain " signifiant qu'il est habitant du pays nouveau et du continent tout entier. Comme le dira Thomas Paine en 1783, l'auteur de Common Sense: * Être citoyen d'un État particulier n'est qu'une distinction de portée locale [...] Notre titre suprême est celui d'Américain, les appellations subalternes different, elles, d'un lieu à l'autre. " 4

Force des choses et force des mots s'affrontent ou se conjoignent dans le discours parlé ou écrit, disais-je dans un texte publié il y a déjà quelque temps 5 . Pourquoi devrais-je me laisser emporter par la dérive du sens que certains voudraient m'imposer par leur facon d'user des mots? Si Thomas Paine reconnaissait lui-même qu'il était citoyen d'un état particulier pourquoi lui reconnaître la légitimité de son appropriation d'un titre suprême? Les États-Unniens qui, à la manière de Napoléon se couronnant lui-même, se sont intronisés dans ce statut suprême ont vite fait de passer à l'échelon supérieur: celui de gendarme du monde. Ainsi on a vu que depuis un certain temps le président des U.S.A. ne fait plus devant le Congrès de son pays un rapport sur «l'état de l'Union * mais sur * l'état du monde *.

\footnotetext{
4 Heinz Weinmann, * Les fantômes de l'Amérique ", Cinémas, p. 37.

5 Maximilien Laroche, " "Pouvoir du noir" de Roland Giguère ou la poésie comme critique de l'idéologie ", dans Deux études sur la poésie et l'idéologie québécoises, coll. * Sciences de la culture ", n 3, Québec, Institut supérieur des sciences humaines, Cahiers de l'ISSH, 1975, p. 25.
} 
Il est donc surprenant que même ceux qui déconstruisent fort bien l'opération de surenchère sémantique qui fait passer d'États-Unien à Américain ne continuent pas moins de reprendre à leur compte le vocabulaire hyperbolique de ce citoyen devenu désormais l'incarnation d'une figure continentale. Ainsi même après moult gloses on ne peut, comme le dit le titre d'un éditorial de Jean-Claude Leblond, qu'être «Å la recherche d'une américanité $*$.

C'est que par le mot *américanité * on essaie de mener une mission impossible: réunir des sens multiples dans un mot univoque. Car si comme l'a fort bien reconnu Thomas Paine, le mot Amérique est suprême et les mots ÉtatsUniens, Canadiens, Haïtiens, Mexicains... subalternes, l'on ne peut parler en général d'une chose particulière, d'une façon suprême d'un individu subalterne. Or désigner un citoyen particulier par ce titre suprême d'Américain ne peut être qu'un coup de force, comme je disais un peu auparavant, la métonymisation survalorisante d'un signifiant, ce qui est le propre de l'hyperbole. Accepter alors cette figure comme naturelle et allant de soi, la banaliser et refuser d'y voir une représentation démesurement élogieuse de soi, c'est prendre pour acquis une inflation de sens.

On peut donc comprendre que Weinmann reconnaisse que "la notion d'américanité est floue, évanescente, à l'image même des fantômes *. Mais comme il ne continue pas moins d'utiliser ce terme évanescent dans le sens convenu, il devient impossible pour lui de sortir du cercle vicieux de cette évanescence. «La notion d'américanité est floue, évanescente..." reconnaît Weinmann. Mais il ajoute: «Et pourtant l'américanité est bien réelle, nous en faisons intimement partie, nous sommes bien des Nord-Américains. * 7

Il faut rompre ce carcan qui unit de force un certain mot et une chose: le mot américanité, par exemple, et la réalité Amérique du Nord et même plus particulièrement ce mot et la réalité U.S.A., comme cela apparaît dans la dernière citation. Il faut donc réinsérer la réalité particulière dans son

6 Jean-Claude Leblond, * Éditorial ", Vie des Arts, Amérique latine: l'art des années quatro-vingt, vol. XXXV1, $n^{\circ} 143$, juin 1991, $p .3$.

7 Heinz Weinmann, loc. cit. 
92

ensemble: la réalité suprême, apprendre à user des mots selon leur équivalence ou égalité de sens et non selon leur rapport hyperbolique, c'est-à-dire déséquilibré, avec les choses.

\section{Qu'est ce que l'Amérique ?}

Et d'abord il faudrait se demander ce qu'est l'Amérique, s'il ne faut pas plutôt parler des Amériques, et à ce momentlà, on pourrait parler d'une américanité des * compagnons des Amériques » et même aller jusqu'à réinventer les Amériques, comme le souhaitaient Bell Gale Chavigny et Gari Laguardia, dans leur livre intitulé précisément: Reinventing the Americas $^{8}$.

C'est qu'en français, on parle presque toujours et uniquement de découverte de l'Amérique. En langue espagnole, on se sert aussi et même plus souvent du mot conquête (la descubierta de America/la Conquista de America). Ce n'est d'ailleurs pas sans raison qu'en français on doit emprunter à la langue espagnole le mot * conquistador * quand on veut parler de conquérants qui ont subjugué les Aztèques, les Mayas et les autres peuples aborigènes de l'Amérique centrale et du Sud.

Mais les Espagnols usent aussi du terme Invencion, l'invention de l'Amérique ${ }^{9}$, s'étant rendu compte que la prétendue découverte, en réalité conquête, n'était finalement qu'une invention. La meilleure preuve étant le fait que nous appelons Indiens des gens qui n'ont rien à voir avec le souscontinent asiatique qu'on appelle Inde. Et c'est dans cette perspective d'une invention et d'une réinvention des Amériques que Bell Gale Chavigny et Gari Laguardia, dans leur livre précédemment cité, pensent que la première opération à effectuer consisterait à démasquer cette incohérence rhétorique, comme ils disent, que nous commettons quand nous disons l'Amérique, à propos des États-Unis.

8 Bell Gale Chavigny, Gari Laguardia, Reinventing the Americas, Comparative Studies of Literature of the United States and Spanish America, Cambridge, Cambridge University Press, 1986.

9 Fernando Ainsa, « L'invention de l'Amérique, signes imaginaires de la découverte et construction de l'Utopie ", Diogéne, n 145, janvier-mars 1989, p. 104-116. 
Reinvention of the Americas must begin with exposure of the rhetorical incoherence we commit each time we designate the United States by the sign * America ", a name that belongs by rights to the hemisphere.... As long as we perpetuate the rhetorical malpractice, we lend linguistic support to imperialist enterprises, and, more to our point here, we confuse our thinking. ${ }^{10}$

Alors il faut redéfinir l'Amérique par les Amériques et la réinventer en passant de la fiction de la découverte à la réalité de la conquête, pour aboutir à la dialectique de sa construction largement à venir encore.

Car être Américain, pour le plus grand nombre des habitants de ce continent, c'est essayer de se défaire, 500 ans après, d'une Histoire qui colle à la peau comme un habit étriqué. C'est donc rêver d'une Histoire à venir, quand l'image de soi, ce que Jacques Stephen Alexis comparait précisément à un habit, sera ajusté à notre taille.

Mais surtout, et donc bien plus que ces mauvais souvenirs et ces rêves d'avenir, être Américain, c'est avoir connu la métamorphose qui change un Européen, un Africain, un Asiatique, et même le premier habitant de cette terre, en cet homme nouveau, véritable habitant d'un Nouveau Monde.

Car tous ceux qui habitent aujourd'hui l'Amérique sont venus d'ailleurs, il y a plus ou moins longtemps et ont subi la métamorphose qu'imposaient cette terre et son histoire, selon les modalités particulières de leur entrée dans ce Nouveau Monde et selon les péripéties de leur existence ici.

Même les Aborigènes ont changé ou doivent changer. Ils nous en donnent d'ailleurs la preuve quand ils adaptent leurs tactiques de combat aux réalités de notre époque et usent de l'image médiatique comme jamais leurs ancêtres n'ont su se servir de leurs flèches. Ils font mouche sur leur cible, aujourd'hui, par une image retransmise ou une entrevue rapportée beaucoup plus sûrement qu'avec leurs tomahawks d'antan.

Qu'est-ce que l'Amérique donc sinon la terre où l'on s'efforce de résoudre non pas simplement une contradiction

Bell Gale Chavigny, op. cit., p. VIII. 
94

mais, comme le disent les Haïtiens, une contradiction marassa, c'est-à-dire jumelle. Ce qui a pour effet non seulement de transformer un homme mais de le métamorphoser.

Si l'on prend l'exemple du schéma actantiel que la sémiotique européenne tient pour le modèle d'explication et de résolution de toute opposition ou contradiction, on s'aperçoit que ce schéma, au fond, ne vaut que pour une transformation matérielle: l'acquisition d'un objet. Ainsi ce schéma ne considère que la transformation qui s'effectue sur un seul plan: je te prends ta fille, ta femme, ta maison, ton terrain, ton ministère, ton gouvernement ou ton pays. À peine peut-on parler de transformation psychologique puisqu'il s'agit avant tout de l'acquisition d'un objet. Par l'intermédiaire d'un désir sans doute: un vouloir-faire, qui s'apparente cependant à son tour à l'appropriation d'un bien matériel.

Mais si je veux changer de plan? Le ver à soie qui veut devenir papillon, l'homme ordinaire qui veut devenir un saint, un héros? Il me faut alors résoudre une double contradiction, une contradiction marassa ou jumelle. Car toute métamorphose s'effectue contre et par un autre en même temps que pour et contre moi-même. Le ver à soie lutte contre sa nature et reçoit son aide pour passer du plan rampant au plan volant. Le futur saint rivalise avec Dieu en même temps qu'il requiert son aide. Et bien sûr le héros tue en lui l'homme ancien pour faire naître l'homme nouveau.

Double combat où l'adversaire est un adjuvant et où le bénéficiaire est un opposant; où des rôles contradictoires sont donc tenus par le même acteur. Or c'est bien cela la situation de l'habitant des Amériques. La manière dont il a toujours associé le politique et le religieux peut le faire voir.

Les Pilgrim Fathers états-uniens sont venus en terre américaine avec un double objectif: religieux et politique. Et les dollars d'aujourd'hui le rappellent toujours. "In God we trust \#, y peut-on lire. L'argent et la foi associés! Mais c'était déjà là ce qui caractérisait l'entreprise de Christophe Colomb qui tout en plantant des croix sur les terres qu'il conquérait lorgnait du côté des mines d'or qu'il pouvait prospecter.

On peut donc sur la base de cette association de la foi et de la politique tenir l'Amérique pour la terre des «vodouns", 
c'est-à-dire des religions syncrétiques qui s'efforcent de faire passer l'individu, et même la collectivité, du plan politique au plan religieux, de métamorphoser l'une de ces dimensions en l'autre.

Le mot «vodoun ", dans les langues de la côte occidentale de l'Afrique, signifie esprit. En Haïti, et dans les formes diverses que prennent les religions syncrétiques afroaméricaines dans la Caraïbe, au Brésil et même en Amérique du Nord, ces cultes afro-américains se caractérisent par la transe et la possession, par le spectacle d'une métamorphose de l'individu, en somme, puisque le fidèle est possédé par un esprit et devient ainsi un autre.

À divers égards c'est ce type de métamorphose qui s'opère dans les cultes afro-états-uniens qui sont à l'origine des gospels, des spirituals, des blues et du jazz. Mais il ne faut pas hésiter à voir dans les preachers de tout acabit, de Billy Graham à Jimmy Swaggart, des « houngans *, c'est-à-dire des prêtres d'une religion de la possession par les esprits, de la métamorphose de l'individu. Sans doute entre un coin reculé de la campagne haitienne ou du terreiro d'une favella de Salvador aux grands stades hypermodernes où prêche Billy Graham ou encore au complexe médiatico-religieux que Jimmy Swaggart gère près de Bâton Rouge, il peut sembler y avoir une distance infranchissable et des différences irréductibles. Il n'en est rien pourtant. L'offrande que fait le fidèle états-unien qui tout de suite après avoir écouté son preacher à la télé lui poste son chèque équivaut aux manje-lwa que le vodouisant va déposer à un carrefour. Ici et là on prie, on chante, on danse et la merveille survient: on est guéri, on est illuminé, la voix de l'esprit se met à parler par notre bouche. Chant, danse, transe. Discours, musique, possession. Le schéma est le même. Et l'objectif aussi: se métamorphoser.

On aurait donc tort d'ironiser sur le croyant qui va au parking suivre de sa voiture une cérémonie religieuse retransmise sur grand écran ou de regarder d'un œil sceptique le fidèle qui fait tournoyer au-dessus de sa tête une poule ou qui dépose une offrande de grains de maïs à un carrefour. Les deux sont en train, avec les moyens du bord et du moment, d'opérer leur métamorphose en ces esprits auxquels ils croient et qui sont aussi physiques que spirituels, 
qui sont dans le ciel ou sur les dollars et peuvent, de la Guinée ou de l'au-delà, venir se loger dans nos têtes.

Si les cultes réformés états-uniens ont tant de succès dans la Caraibe et en Amérique latine ce n'est pas tellement à cause de la puissance politique et commerciale des ÉtatsUnis mais surtout parce que la Bible joue pour les uns comme pour les autres, pour tous les Américains, le même rôle. Car ce n'est pas uniquement pour l'analphabète caraỉbe ou latinoaméricain mais pour tous les Américains que le livre sacré joue le rôle de convertisseur merveilleux de l'écrit en oral.

Entre Luther et les docteurs de l'Église catholique de son temps, il se déroulait un formidable combat de doctes. Billy Graham ou Jimmy Swaggart sont des docteurs de leur propre autorité. De celle de leur charisme et de leur rhétorique. Comme le houngan qui est son propre patron, dans son hounfort. Pour parler en termes vraiment irrévérencieux, les uns et les autres sont les proprios de leur boîte.

Si l'on s'efforçait de considérer un hounfort de ce strict point de vue organisationnel, du management disons, on y verrait un modèle d'entreprise privée. Et si bien sûr en Haïti, à Cuba et au Brésil, on ne peut lancer qu'une P.M.E., il va de soi qu'aux U.S.A. on commence d'emblée par fonder une multinationale.

Cela est dans l'ordre des choses. L'ordre laïque donne la forme et le mode d'articulation du profane au religieux dans un espace donné. Or, pour en revenir à Christophe Colomb et aux Pilgrim Fathers, nous savons que le premier venait chercher ici l'or et les épices de l'Asie. Il venait faire des affaires mais en se couvrant du manteau de la religion. Quant aux Pères fondateurs des États-Unis, ils fuyaient la persécution religieuse et venaient donc bâtir ici la nouvelle Jérusalem. Mais ils ont vite fait de passer à l'expropriation des terres, à la colonisation et à l'esclavage. Ils sont revenus au «business as usual ", pourrait-on dire.

Une illusion est malgré tout demeurée au travers de tous les avatars de ces diverses formes de découvertes-conquêtes de l'Amérique: celle de pouvoir opérer une métamorphose de l'homme. Illusion, dans la mesure où la métamorphose souhaitée doit être réalisée volontairement, planifiée même et exécutée et non pas subie, consentie ou acceptée tout simplement. 
Peut-on lutter contre Dieu et en même temps recevoir son aide? Sans doute. « Soyez parfaits comme votre père céleste", nous dit le Christ. Toute comparaison est mesure et forme d'une certaine lutte. Mais la comparaison est aussi proposition d'un modèle. Il s'agirait donc de rivaliser avec Dieu en acceptant de se soumettre à ses conditions. Cette rivalité est une émulation pour l'homme. On voit réapparaître la contradiction jumelle dont je parlais déjà comme condition de la métamorphose de l'Américain.

Sans entrer dans des querelles de théologien et pour ne rester que sur le terrain de la contradiction de l'oral et de l'écrit, on peut dire que la Parole de Dieu, parce que nous ne la connaissons que sous sa forme écrite, fixée et immuable, est une. Mais comme nous devons, chacun de nous, l'appliquer, elle redevient orale, multiple. Comment concilier l'écriture de Dieu et notre oralité ? Comment de l'écriture de Dieu passer par notre oralité pour retrouver l'oralité de la parole divine, c'est-à-dire son caractère non contradictoire d'être à la fois une et multiple?

Partir de la parole de Dieu ( In God we trust») pour arriver, en passant par sa propre parole ( Thous les hommes naissent et demeurent libres et égaux en droit»), à la parole de Dieu à la fois adaptée à notre situation et valable pour tous les hommes, c'est là la gageure. Et on pourrait la représenter par le parcours d'une écriture qui, adaptée à notre oralité, permettrait d'aboutir à une écriture-oralité universelle.

On parle de rêve américain et on l'assimile à tort au rêve états-unien qui n'en est qu'une manifestation. Ce rêve américain, il est plutôt là, dans cette entreprise qui veut associer le religieux au politique pour aboutir à la résolution de notre contradiction jumelle, celle de devoir être un nouvel Adam, comme on en a toujours rêvé dans la littérature états-unienne, ou d'être un zombi qui aurait goûté au sel de la vie, comme ne cessent de l'espérer les écrivains haïtiens.

Ce rêve américain, si on le comprend ainsi, nous fait mieux comprendre la paradoxale, surprenante, bizarre même, figure du Magoua qu'évoque Jacques Ferron. Ce rêve américain nous fait comprendre aussi en quoi le rêve québécois, à sa manière, est aussi partie intégrante du rêve américain. Ce 
qui éclaire par le fait même la position singulière de Jacques Ferron dans la tradition littéraire québécoise.

On sait que Ferron a parlé de la religion des Québécois comme d'un vodoun. Et c'est dans cette perspective qu'il a donné le titre de Papa Boss à l'un de ses romans, voulant par homophonie faire songer à Papa Doc Duvalier et à tout ce qu'on pouvait imaginer de lui. Dans Le ciel de Québec et sur le modèle cette fois de la mythologie gréco-romaine, il a tenté de brosser le panorama d'une mythologie bien locale par ses personnages et ses enjeux. Mais c'est finalement dans Le Saint-Élias qu'il a esquissé cette figure du Magoua, un personnage québécois qui serait l'équivalent du nouvel Adam états-unien ou du zombi haïtien. Croisement d'Européen et d'Amérindien, de notable et de quêteux, en somme synthèse ou syncrétisme de toutes les contradictions québécoises, le Magoua est au fond tout simplement le Québécois tel qu'en lui-même il se métamorphosera, selon Ferron, évangéliste du Québec.

L'entreprise de peindre ce Québécois métamorphosé en lui-même était trop volontaire chez Ferron pour être toujours convaincante. Et c'est peut-être une des raisons pour lesquelles elle a été peu soulignée jusqu'à présent, étant passée à peu près inaperçue. Mais cet aveuglement de la critique est peut-être significatif des obstacles qu'affrontait Ferron.

Amérique, terre des métamorphoses! Par le rêve des hommes ou la force des choses et de l'Histoire, cet espace est destiné à voir les hommes venus d'outre-mer se métamorphoser. Car même les dits Autochtones ne sont-ils pas arrivés ici, les uns en enjambant le détroit de Behring pour passer en Alaska tandis que d'autres empruntaient plutôt le passage de la Polynésie à la terre de Feu? Pour se métamorphoser donc, et non pas simplement en acquérant des objets: de l'or, des terres, mais en changeant d'être.

Les États-Unis ne sont pas toute l'Amérique. Loin de là! Pourtant la prétention si peu contestée des Etats-Uniens de se faire appeler Américains pourrait bien souligner le fait qu'ils sont peut-être, à l'heure actuelle, ceux qui sont le plus près de réaliser un certain rêve américain. Ne sont-ils pas parvenus à croire eux-mêmes à l'illusion qu'on puisse être un " self-made man " et à la rendre crédible pour les autres? Or 
comment pourrait-on " s'être fait soi-même * quand on n'est même pas capable de *se faire soi-même*, d'être l'auteur et le seul possesseur de ses propres jours? Métamorphose, quand ton fantasme nous tient! 